\title{
Monochromatic green light stimulation during incubation shortened the hatching time via pineal function in White Leghorn eggs
}

Panlin Wang ${ }^{1,2+}$, Yanyan Sun ${ }^{1 \dagger}$, Yunlei Li ${ }^{1}$, Jing Fan', Yunhe Zong ${ }^{1}$, Adamu Mani Isa ${ }^{1}$, Lei Shi ${ }^{1}$, Yuanmei Wang ${ }^{1}$, Aixin $\mathrm{Ni}^{1}$, Pingzhuang $\mathrm{Ge}^{1}$, Linlin Jiang ${ }^{1}$, Shixiong Bian ${ }^{1}, \mathrm{Hui} \mathrm{Ma}^{1}$, Zhengdong Yuan ${ }^{3}$, Xiaolin $\mathrm{Liu}^{2^{*}}$ and Jilan Chen ${ }^{1 *}$ (D)

\begin{abstract}
Background: Effect of monochromatic green light illumination on embryo development has been reported in chickens. The avian pineal gland is an important photo-endocrine organ formed by a mediodorsal protrusion during embryonic development. However, the involvement of pineal gland in the light transduction process remains to be elucidated. In the present study, we investigated the influence of monochromatic green light on hatching time and explored the possible mechanism via pineal function.
\end{abstract}

Results: A total of 600 eggs of White Leghorn (Shaver strain) were incubated under photoperiods of either $12 \mathrm{~h}$ of light and $12 \mathrm{~h}$ of darkness using monochromatic green light (12L:12D group) or $24 \mathrm{~h}$ of darkness (OL:24D group) for $18 \mathrm{~d}$. Compared to OL:24D group, the green light stimulation shortened the hatching time without extending the hatch window or impairing hatchability. The liver of embryos incubated in the 12L:12D light condition was heavier than those of the OL:24D group on d 21 post incubation which may be linked to the observed increase in the serum concentration of insulin-like growth factor 1 (IGF-1); primary secretion of the liver. Histological structure analysis of pineal gland demonstrated that the light stimulation increased follicle area, wall thickness and lumen area on $\mathrm{d} 10$ and $\mathrm{d} 12$ post incubation. Rhythmic function analysis demonstrated that three clock related genes (brain and muscle ARNT-like-1, BMAL1; circadian locomotor output cycles kaput, CLOCK; and cryptochrome-1, CRY1) and a melatonin rate-limiting enzyme related gene (arylalkylamine $\mathrm{N}$-acetyltransferase, AANAT) were rhythmically expressed in the pineal gland of the 12L:12D group, but not in the OL:24D group. Simultaneously, the light stimulation also increased the concentration of melatonin (MT), which was linked to hepatocyte proliferation and IGF-1 secretion in previous studies.

(Continued on next page)

\footnotetext{
* Correspondence: liuxiaolin@nwsuaf.edu.cn; chen.jilan@163.com

${ }^{\dagger}$ Panlin Wang and Yanyan Sun contributed equally to this work.

${ }^{2}$ College of Animal Science and Technology, Northwest A\&F University,

Yangling 712100, China

'Institute of Animal Sciences, Chinese Academy of Agricultural Sciences, Key

Laboratory of Animal (Poultry) Genetics Breeding and Reproduction, Ministry

of Agriculture and Rural Affairs, Beijing 100193, China

Full list of author information is available at the end of the article
}

C C The Author(s). 2021 Open Access This article is licensed under a Creative Commons Attribution 4.0 International License, which permits use, sharing, adaptation, distribution and reproduction in any medium or format, as long as you give appropriate credit to the original author(s) and the source, provide a link to the Creative Commons licence, and indicate if changes were made. The images or other third party material in this article are included in the article's Creative Commons licence, unless indicated otherwise in a credit line to the material. If material is not included in the article's Creative Commons licence and your intended use is not permitted by statutory regulation or exceeds the permitted use, you will need to obtain permission directly from the copyright holder. To view a copy of this licence, visit http://creativecommons.org/licenses/by/4.0/ The Creative Commons Public Domain Dedication waiver (http://creativecommons.org/publicdomain/zero/1.0/) applies to the data made available in this article, unless otherwise stated in a credit line to the data. 
(Continued from previous page)

Conclusions: The 12L:12D monochromatic green light stimulation during incubation shortened hatching time without impairing hatching performance. Pineal gland's early histological development and maturation of its rhythmic function were accelerated by the light stimulation. It may be the key organ in the photo-endocrine axis that regulates embryo development, and the potential mechanism could be through enhanced secretion of MT in the 12L:12D group which promotes the secretion of IGF-1.

Keywords: Circadian rhythm, Hatching time, Insulin-like growth factor 1, Melatonin, Monochromatic green light, Pineal gland

\section{Background}

Avian species have a broader visible light spectrum [1, 2] and special extra-retinal photoreceptor in pineal gland $[2,3]$. They are more sensitive to environmental lighting than mammals. In commercial poultry hatcheries, fertile eggs are incubated in complete darkness, which is different from the settings in natural incubation. There are evidences that light can increase both body weight and breast muscle weight at the embryonic and posthatching periods $[4,5]$. Furthermore, monochromatic green light was shown to promote the growth of spleen [6] and skeletal muscle [7] in broilers. Additionally, some studies have reported that light stimulation using light emitting diode (LED) during embryogenesis shortened the hatching time $[8,9]$. The stimulating effect of monochromatic green light on skeletal muscle was linked to enhanced circulation levels of insulin-like growth factor 1 (IGF-1) in blood [7, 10, 11]. Classically, IGF-1 is a ubiquitous peptide secreted mainly in the liver and has been tied to growth hormones, and considered as a surrogate marker for overall growth hormone status [12]. IGF-1 may therefore play an important role in the lightmediated advanced development of chick embryo.

The avian pineal gland is a derivative of the posterior roof of the diencephalon, and a photo-endocrine organ which plays key role in transducing light information to whole body physiology. It was reported that environmental factors could alter the morphology of the pineal gland. For example, great variations in pinealocyte architecture were observed between nocturnal and diurnal birds [13]. Largest pineal gland was recorded in newborn South Pole seals [14], and it contributes to survival in the harsh environment [15]. Pineal gland reflects a high degree of environmental adaptive plasticity not only in histology but also in rhythmic function [13]. Clockrelated genes, including brain and muscle ARNT-like-1 (BMAL1), circadian locomotor output cycles kaput (CLOCK), and cryptochrome-1 (CRY1), and the melatonin (MT) rate-limiting enzyme related gene of arylalkylamine $\mathrm{N}$-acetyltransferase $(A A N A T)$ played crucial roles in establishing circadian rhythm within pineal cells and showed rhythmic oscillations under light-dark cycles in adult chickens [16]. Thus far, our knowledge about the histology and rhythmic function of pineal gland during embryogenesis in chickens is still insufficient, especially the comparison effects under various light treatments. Above all, whether the pineal gland is involved in light transduction process during embryogenesis and its potential link with IGF-1 is still unknown.

The objective of this study was to investigate the effect of 12L:12D monochromatic green light stimulation during embryogenesis on hatching time and hatching performance in White Leghorn (Shaver strain), and explore the possible mechanism with the involvement of pineal gland.

\section{Materials and methods \\ Experimental design}

A total of four incubators (NK-hatching; Beili Incubation Equipment Co. Ltd., Sichuan, China) used in the experiment were blacked out with shade cloth. Two were outfitted with monochromatic green LED strips (ND-BIS2D-D10W, Nodark Biolight Technology Co., Ltd., Wuxi, China) $(\lambda=520-525 \mathrm{~nm})$ while the other two were left with no light source. Fertile eggs were obtained from White Leghorn layers (Shaver strain) of 50 weeks of age. The pure line chickens were sourced from the University of Guelph, Canada and housed in the experimental farm of the Institute of Animal Sciences, Chinese Academy of Agricultural Sciences, Beijing, China. A total of 600 eggs of normal size with an average weight of $62.5 \pm 3.0 \mathrm{~g}$ were selected and stored for no longer than $7 \mathrm{~d}$ at $15^{\circ} \mathrm{C}$ and $70-75 \%$ relative humidity. Eggs were randomly allocated to the two groups, with two incubators (replicates) per group, and 150 eggs per incubator. Eggs of one group were incubated in monochromatic green lightfitted incubators with a light:dark schedule of 12L:12D and light intensity of $200 \mathrm{~lx}$ during the first $18 \mathrm{~d}$ of incubation, namely 12L:12D group. Eggs in the control group were incubated in complete darkness and named 0L:24D group. The temperature and humidity were calibrated by a standard thermometer and hygrometer for the four incubators before incubation and monitored every $2 \mathrm{~h}$ during the whole incubation period. The incubation was maintained at a temperature of $37.8 \pm 0.10{ }^{\circ} \mathrm{C}$, and a relative humidity around $60 \%$ until d 18 . The incubator 
temperature changes on $\mathrm{d} 7$ post incubation were showed as an example (Additional file 2: Fig. S1.). From d 19, eggs were transferred to hatching baskets and a temperature of $37.2 \pm 0.10{ }^{\circ} \mathrm{C}$ and relative humidity of $70 \%$ was set for the hatcher. At the end of embryonic d $17,18,19,20$, and 21 , three embryos or chicks were randomly selected and weighted from each replicate (incubator). The hearts, livers, and pectoral musscles were isolated and weighed to track the embryo and organ development. The weight was calculated using the average weight of each replicate for the statistical analysis.

\section{Hatching time and hatching performance}

From d 19 post incubation, all eggs were transferred to the same hatcher (Haijiang Hatching Equipment Co., Ltd., Beijing, China) in constant darkness until hatching. The number of hatched chicks was counted every $2 \mathrm{~h}$ from $468 \mathrm{~h}$ to $512 \mathrm{~h}$ post incubation to monitor the hatching time for each egg and assigned to the nearest time of hatch. Average hatching time, time to $90 \%$ hatch, peak hatching period, and hatch window were recorded for each replicate. Average hatching time was calculated as the mean duration from egg setting to the emergence of chicks for all hatched chicks in a given batch. Time to $90 \%$ hatch was the hatching time that $90 \%$ of the chicks hatched. Peak hatching period was defined as the duration that $30 \%$ to $70 \%$ chicks hatched [17]. Hatch window was defined as the time interval from the emergence of the first chick to the last chick [17, 18]. Hatchability was calculated as a percentage of fertile egg number. Every hatched chick was assessed for chick quality within 4-6 h after hatching according to a standardized method of scoring in a scale of 100 [19].

\section{Histology of pineal gland}

To explore the possible mechanism that monochromatic green light affects hatching time, another hatching trial was performed using the same incubation conditions and light treatments. Pineal glands were extracted from six birds each, in the 12L:12D and 0L:24D groups on $d$ $10,12,14,16$, and 18 post incubation. The tissues were immediately fixed in $10 \%$ neutral buffered formalin. On the 7 th day after fixation, all samples were dehydrated in a graded series of alcohols, embedded in paraffin, and dissected into $4 \mu \mathrm{m}$ thick. These sections were stained with Harris haematoxylin and eosin for histomorphology observation using a Zeiss Axioskop microscope (Carl Zeiss, Thornwood, NY, USA) equipped with a QICam digital camera and NIS Elements software (Nikon Instruments, Melville, NY, USA). The pineal follicle related indicators, including wall thickness (WT), follicle (FL) area, and lumen (LM) area were measured using Digimizer Image Analysis software (Ostend, Belgium).

\section{Expression of clock-related and AANAT genes in the pineal gland}

The expression of clock-related genes (BMAL1, CRY1 and $C L O C K$ ) and the melatonin rate-limiting enzyme related gene $(A A N A T)$ in the pineal gland was measured using real-time quantitative RT-PCR. Pineal glands were collected every $4 \mathrm{~h}$ over a 28 -h period from d 17 to $\mathrm{d} 18$ after incubation (zeitgeber time (ZT) 2, 6, 10, 14, 18, 22, and 26, where ZT0 is the time when the light is turned on, ZT12 is the time when the light is turned off). Pineal glands removed during dark period were obtained from birds euthanized and decapitated under dim red light. Six pineal glands were collected at each time point for each group, and the sampling time of $0 \mathrm{~L}: 24 \mathrm{D}$ group was synchronized with those of 12L:12D group. Two pineal glands were pooled and the total RNA of three pooled samples for each group were extracted with TRIzol reagents following the manufacturer's protocol (Tiangen Biotech, Beijing, China). cDNA was generated by reverse transcription using $1 \mu \mathrm{g}$ total RNA in a total of $20 \mu \mathrm{L}$ reaction volume following the instruction of the manufacturer (TaKaRa, Shiga, Japan). Primer 6.0 was used to design primer sequences for the target genes (Additional file 1: Table S1). Real-time PCR amplification was performed using $1.5 \mu \mathrm{L}$ cDNA solution per $10 \mu \mathrm{L}$ reaction volume with $\mathrm{SYBR}^{\circ}$ FAST qPCR Kit Master Mix (Kapa Biosystems, Wilmington, USA). The relative expression of target genes was calculated using glyceraldehyde-3-phosphate dehydrogenase (GAPDH) as an endogenous control by the $2^{-\Delta \Delta C(T)}$ method [20]. Samples were run in triplicate.

\section{Serum concentrations of melatonin and IGF-1}

Serum concentrations of MT and IGF-1 on d 19 post incubation were analyzed. Circadian time (CT) is a standard marker of time that is based upon the oscillation or rhythm in constant darkness. CT0 and CT12 correspond to the beginning time of the subjective day and night respectively and CT26/2 is the time point of CT2 on the second day. The blood samples of twelve embryos were collected from allantois vein after the birds were euthanized by cervical dislocation every $4 \mathrm{~h}$ from CT2 to CT26/2. The sampling time of embryos was synchronized in the treatment and control groups. Serum was decanted after $2 \mathrm{~h}$ of collection at room temperature. Serum of four embryos were pooled and three pools from each group were subjected to MT and IGF-1 hormonal assay using a commercial ELISA kits (Horabio Biotechnology Co. Ltd., Shanghai, China).

\section{Statistical analysis}

The data of related characteristics of hatching time, hatching performance, pineal follicle histological features, serum hormones, and embryo growth were 


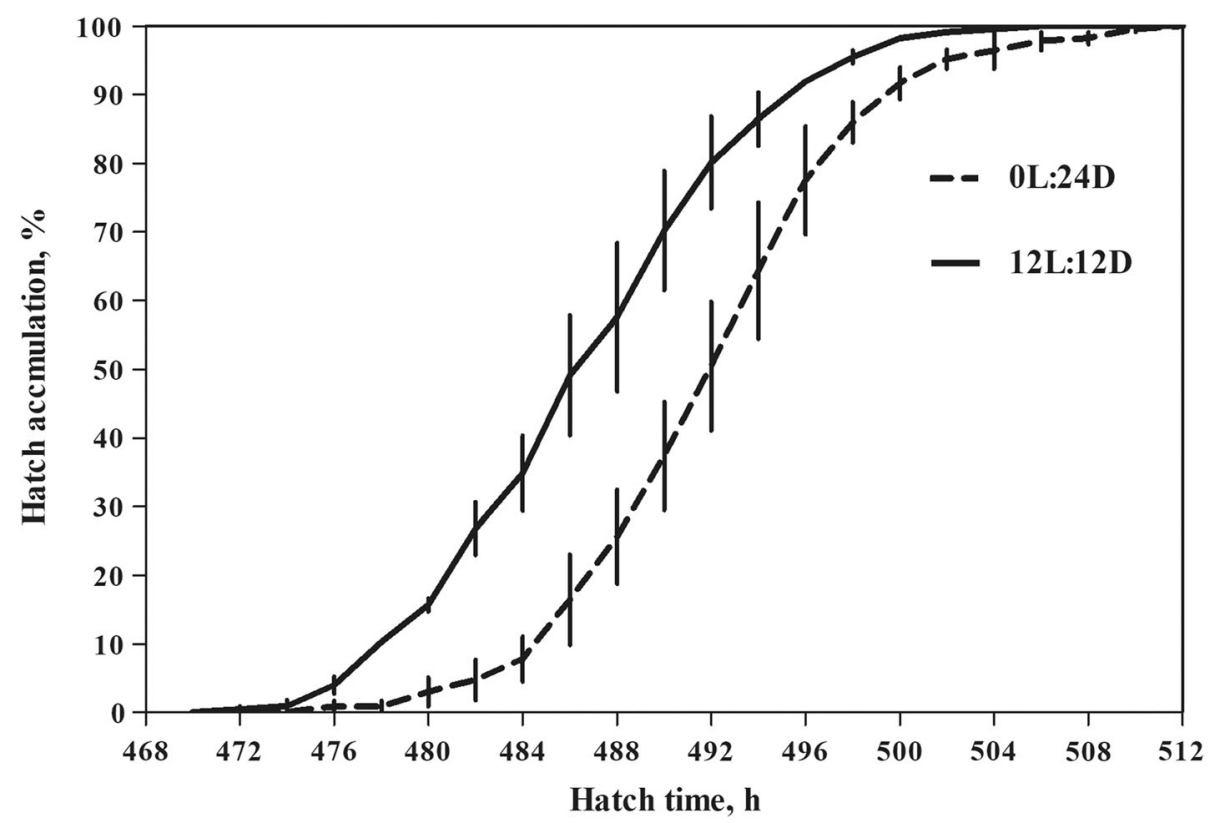

Fig. 1 Hatch accumulation in OL:24D and 12L:12D groups. Data are presented as mean \pm SEM ( $n=2$ per group)

analyzed using $t$-test using SAS software (SAS 9.2, SAS Institute Inc., Cary, NC, USA). All data in percentage were arcsine-transformed before analysis. Threshold for significance of difference was set at $P<0.05$. The rhythm of genes was analysed by GraphPad Prism software using the cosinor formula $y=c+a \times \cos [2(t-\varnothing) / 24]$, where $c, a$ and $ø$ denote the mesor, amplitude and phase of the cosine wave, $t$ is time in hours, and $\mathrm{R}^{2}$ is the fitting degree. Statistically significant differences in gene rhythms were indicated by $P<0.05$ and calculated using the website https://www.danielsoper.com/statcalc/calculator.aspx?id= $15[21]$.

\section{Results}

\section{Hatching time and hatching performance}

The distributions of hatching time are shown in Fig. 1. The 12L:12D group was found to give the first hatching. As shown in Table 1, average hatching time and time to 90\% hatch were shorter in 12L:12D group as compared with $0 \mathrm{~L}: 24 \mathrm{D}$ group $(P=0.04 ; P=0.04$ respectively). However, hatch window and peak hatching period were not affected by the light stimulation $(P=0.29 ; P=0.70$, respectively). Similarly, hatchability and chick quality score were not affected by light treatment $(P=0.72 ; P=0.61$, respectively).

\section{Embryo and organs development}

The weight of embryo, liver, heart and pectoral muscle at different incubation stages are shown in Fig. 2. The results indicate that the light treatment had no effect on the weight of embryo (Fig. 2a), heart (Fig. 2c) or pectoral muscle (Fig. 2d) from d 17 to 21 post incubartion $(P>$ $0.05)$. However, increase in liver weight was observed on d 21 post incubation of the 12L:12D group $(P=0.01)$ (Fig. 2b). Liver, heart, and pectoral muscle weight increased as embryo aged, while embryo weight dropped on $\mathrm{d} 21$.

\section{Histology of pineal gland}

To investigate the effects of monochromatic green light on the structure of pineal gland during embryogenesis,

Table 1 Effects of light treatments on hatching time and hatching performance

\begin{tabular}{|c|c|c|c|c|}
\hline \multirow[t]{2}{*}{ Item } & \multicolumn{2}{|c|}{ Light treatment } & \multirow[t]{2}{*}{ SEM } & \multirow[t]{2}{*}{$P$-value } \\
\hline & OL:24D & 12L:12D & & \\
\hline Average hatching time ${ }^{1}, \mathrm{~h}$ & $492.92^{a}$ & $487.58^{b}$ & 1.60 & 0.04 \\
\hline Time to $90 \%$ hatch $^{2}, \mathrm{~h}$ & $500.00^{a}$ & $494.50^{b}$ & 1.65 & 0.04 \\
\hline Peak hatching period ${ }^{3}, \mathrm{~h}$ & 6.50 & 6.75 & 0.24 & 0.70 \\
\hline Hatch window ${ }^{4}, \mathrm{~h}$ & 33.00 & 31.00 & 0.82 & 0.29 \\
\hline Hatchability ${ }^{5}, \%$ & 84.81 & 85.43 & 0.65 & 0.72 \\
\hline Chick quality score $^{6}$ & 97.01 & 98.02 & 0.75 & 0.61 \\
\hline
\end{tabular}

Data are the mean of two replicates

$\mathrm{a}, \mathrm{b}$ Values within a row with different superscripts differ significantly at $P<0.05$

${ }^{1}$ Average hatching time was calculated as the mean duration from egg setting to the emergence of chicks for all hatched chicks in a given batch

${ }^{2}$ Time to $90 \%$ hatch was the hatching time that $90 \%$ of the chicks hatched

${ }^{3}$ Peak hatching period was defined as the duration that $30 \%$ to $70 \%$ chicks hatched

${ }^{4}$ Hatch window was defined as the time interval from the emergence of the first chick to the last chick

${ }^{5}$ Hatchability was calculated as a percentage of fertile egg number

${ }^{6}$ Chicks were scored for their activity, appearance (plumage, eyes, and legs), and navel area (cicatrisation, retracted yolk, remaining membrane, and yolk) within a total score of 100 [19] 


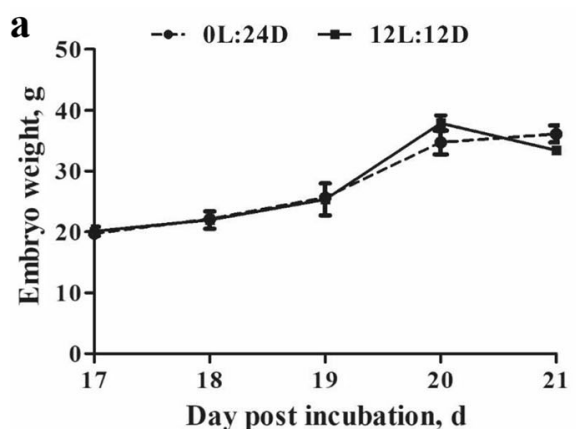

c

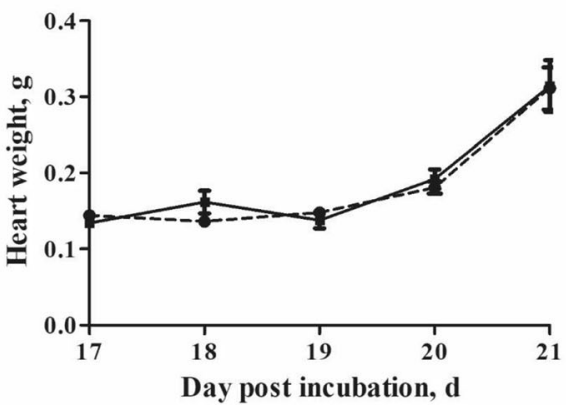

b

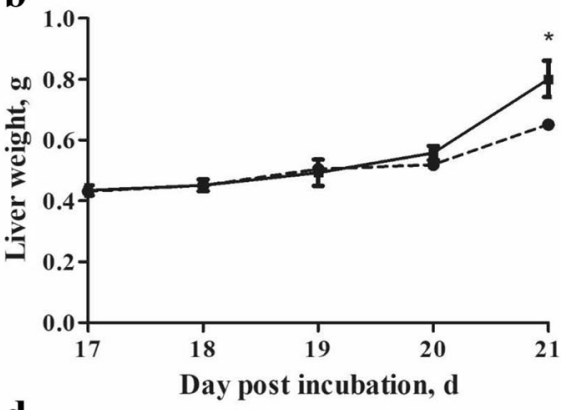

d

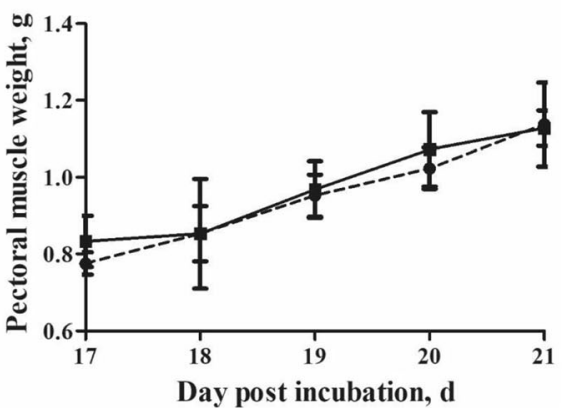

Fig. 2 Effect of light treatment on the weight of embryo (a), liver (b), heart (c), and pectoral muscle (d) from d 17 to 21 post incubation. Asterisk indicates significant difference between groups at a given incubation stage $(* P<0.05)$. Data are presented as mean \pm SEM $(n=2$ per group)

histological observations were performed every 2 days from d 10 to 18 post incubation. The morphological structure on $\mathrm{d} 10,14$, and 18 post incubation are showed in Fig. 3. Follicle area and wall thickness increased with advancing embryogenesis, while lumen area increased from $\mathrm{d} 10$ to 14 post incubation, but decreased thereafter in both groups (Fig. 4). The 12L:12D monochromatic green light stimulation increased follicle area, wall thickness, and lumen area on $\mathrm{d} 10$ and 12 post incubation $(P<0.01)$. Moreover, $\mathrm{d} 18$ post incubation also witnessed thicker walls in the 12L:12D group $(P=0.04)$.

\section{Expression of clock-related and AANAT genes in the pineal gland}

Transcripts of the melatonin rate-limiting enzyme related gene $(A A N A T)$ and three clock-related genes (BMAL1, CLOCK, and CRY1) displayed day-night variations from d 17 to 18 post incubation in both groups (Fig. 5). The cosinor analysis showed that AANAT, $B M A L 1, C L O C K$, and CRY1 exhibited significant circadian rhythm in the $12 \mathrm{~L}: 12 \mathrm{D}$ group $(P=0.00 ; P=0.01$; $P=0.00 ; P=0.01$, respectively), but not in the $0 \mathrm{~L}: 24 \mathrm{D}$ group $(P>0.05)$.

\section{MT and IGF-1 hormones}

Compared with the 0L:24D group, a sharp change of MT levels was observed in the 12L:12D group over the $28 \mathrm{~h}$ period of the hormonal monitoring (Fig. 6a). MT levels at subjective night were higher than those at subjective day in the 12L:12D group $(P=0.00)$, but there was no significant diurnal difference in the $0 \mathrm{~L}: 24 \mathrm{D}$ group $(P=0.24)$ (Fig. 6c). At subjective night, MT levels were higher in the 12L:12D group than those in the 0L: 24D group $(P=0.02)$, but there was no significant difference at subjective day between two groups $(P=0.22)$. Additionally, relatively higher levels of IGF-1 over the $28 \mathrm{~h}$ period were observed in 12L:12D group (Fig. 6b). Furthermore, the IGF-1 levels were higher in the 12L: $12 \mathrm{D}$ group than those in the 0L:24D group at both subjective day and night $(P=0.00 ; P=0.04$, respectively) (Fig. 6d).

\section{Discussion}

In this study, the effect of 12L:12D of monochromatic green light on hatching time and hatching performance of White Leghorn chickens were determined and the possible underling mechanism involved with the pineal gland was investigated.

In order to evaluate the impact of the monochromatic green light on hatching time, average hatching time and time to $90 \%$ hatch were compared. Overall, monochromatic green light stimulation shortened the hatching time. Similarly, Tong et al. [9] reported a 3.4-h shortened hatching time in broilers under monochromatic green LED light stimulation. Decreased hatching time was earlier linked to increase in hatching weight, differences in thyroid hormone levels, organ weight and maturity, post-hatching growth, feeding and general 


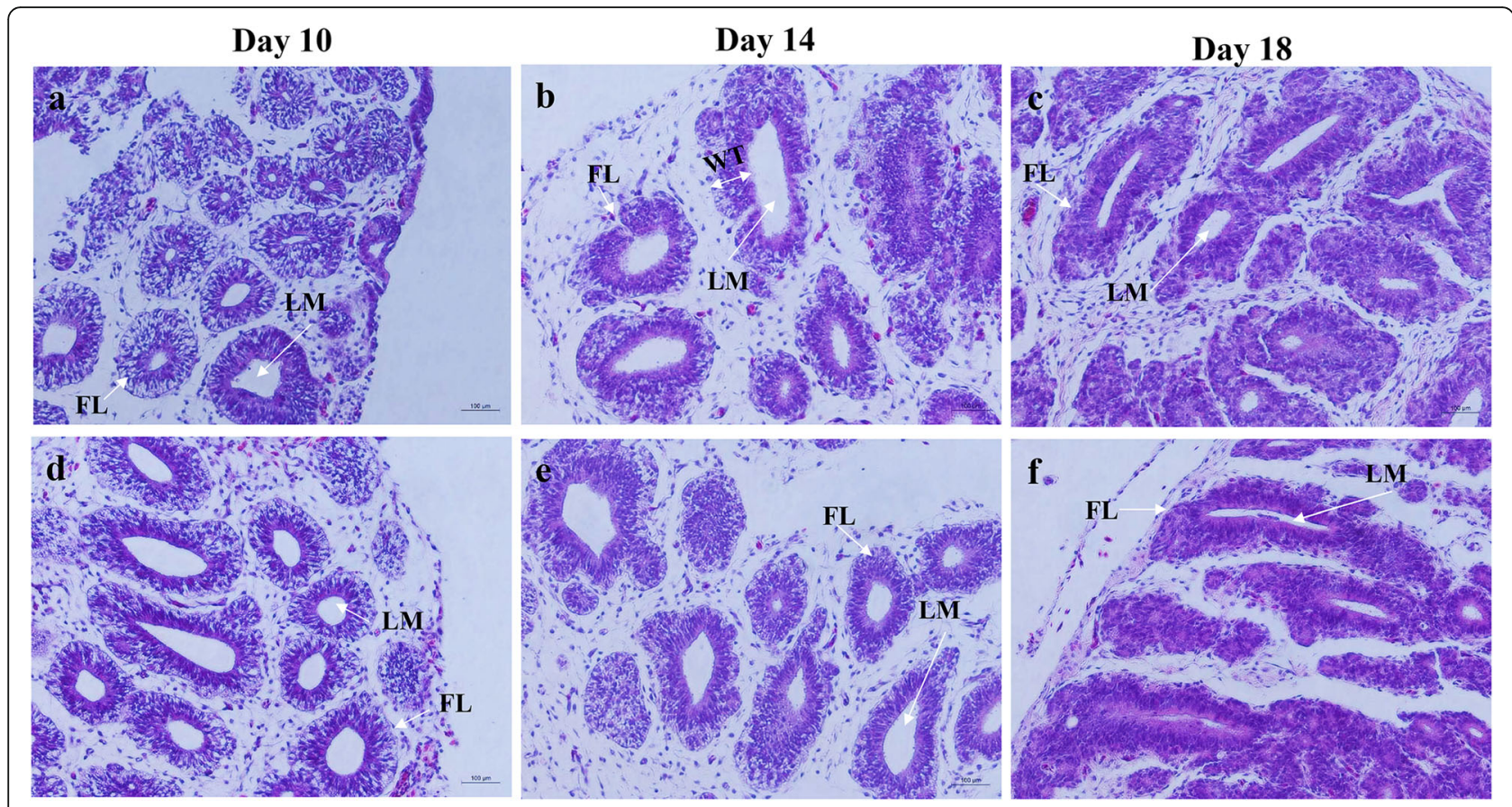

Fig. 3 Histological structure of embryonic pineal gland on d 10 (a), 14 (b) and 18 (c) post incubation in the 0L:24D group d, e and $\mathbf{f}$ represented the counterpart in the 12L:12D group. FL, follicle; LM, lumen; WT, wall thickness.

behavior $[19,22]$. Additionally, shortening the hatching time may result in energy conservation and reduced cost of hatching. However, reduced hatching time is not always beneficial if the hatching period is wider because this subjects early hatched chicks to longer feed and water deprivation $[18,23,24]$. It has been reported that layer chicks with $48 \mathrm{~h}$ feed and water deprivation had a lower body weight and decreased concentrations of serum glucose, total protein, and triglycerides up to $56 \mathrm{~d}$ [25]. Furthermore, scattered hatching period may impair day-old chick quality and post-hatch performance [26]. Hatch window and peak hatching period, representing the intervals and concentration of hatching time of a batch of hatched chicks respectively, were not affected by the light stimulation during embryogenesis as observed in the present study. The hatch window was less than $34 \mathrm{~h}$ in both the treatment and the control groups. Meanwhile, the external light stimulation did not impair hatchability and chick quality. Taken together, the monochromatic green light shortened the hatching time without impairing hatching performance.

Unlike in mammals, avian pineal gland is an important component of the photo-endocrine system containing both photoreceptors and circadian oscillators. The chicken pineal gland during embryogenesis, in contrast to the post-hatching period, was characterized by a
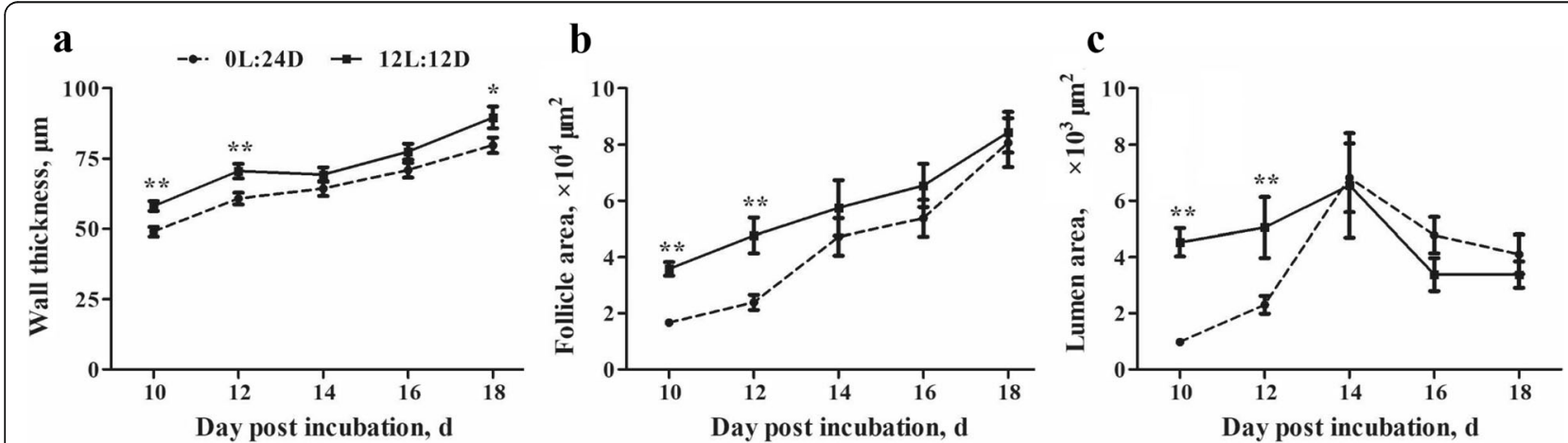

Fig. 4 Effect of monochromatic green light stimulation on the histological structure of pineal gland follicle related indicators including wall thickness $(\mathbf{a})$, follicle area $(\mathbf{b})$, and lumen area $(\mathbf{c})$ from d 10 to 18 post incubation. The data are presented as mean \pm SEM $\left(n=6\right.$ per group). ${ }^{*} P<$ $0.05 ;{ }^{* *} P<0.01$ 
a

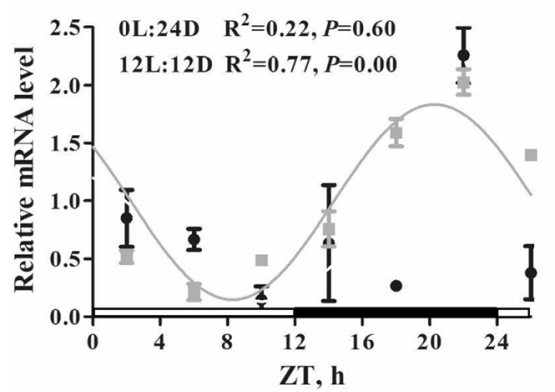

c

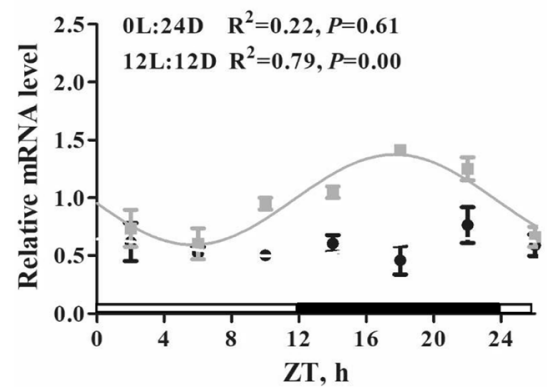

b

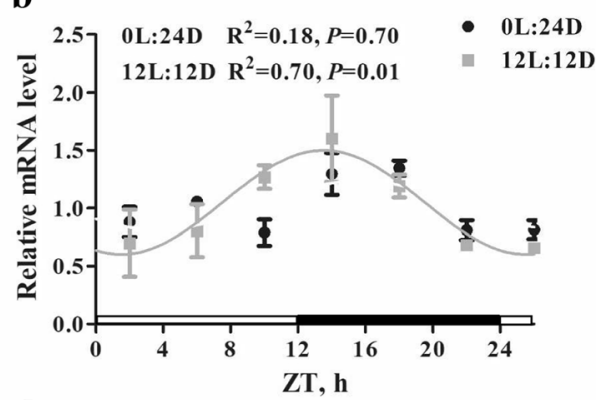

d

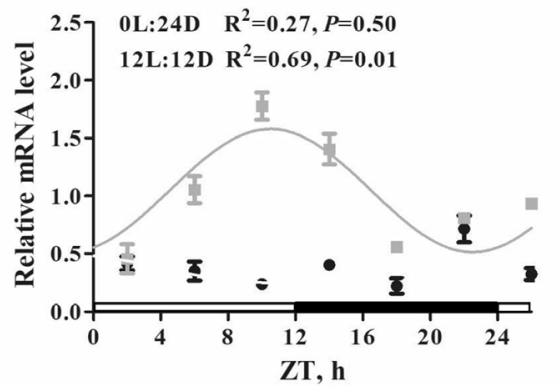

Fig. 5 Temporal changes of mRNA levels of target genes in the pineal gland. a, arylalkylamine N-acetyltransferase (AANAT); b, brain and muscle ARNT-like-1 (BMAL1); c, circadian locomotor output cycles kaput (CLOCK); d cryptochrome-1 (CRY1). The horizontal white bar of the X-axis represents light condition, and the black bar represents dark condition in 12L:12D group, while 0L:24D group is in constant darkness throughout the whole day and it is not shown in the figure. The data are presented as mean \pm SEM ( $n=3$ per group). The curve shows the best fit to the points by cosinor analysis. The solid line represents rhythmical gene expression, and no solid line represents arrhythmical gene expression. $R^{2}$ values represent the fitting degree. $P$ values indicate the significance of cosinor regression analysis. Significance of difference was set at $P<0.05$. ZT, zeitgeber time

follicular structure [27]. Previous histological study demonstrated that the follicle and its lumen markedly varied in size as well as its wall thickness during embryogenesis [28]. Thus far, the pineal histological changes during embryogenesis in chickens are still unclear, especially for embryos incubated with light stimulation. In the present study, we observed a trend that follicle area and wall thickness increased with advancing embryogenesis, while lumen area increased on d 10 to 14 post incubation and decreased thereafter. This is similar with the reports of previous studies that linked larger follicle areas, thicker walls and limited lumens of pineal gland with advancement in embryonic development in chickens [28, 29]. In this study, conspicuous structural differences observed in embryos under light stimulation were found on $\mathrm{d} 10$ and 12 post incubation. In a recent investigation, Petrusewicz-Kosińska et al. [30] reported an increase in follicle size and wall thickness of pineal gland which were asserted to be a product of proliferation of the cells which create inner and outer parts of follicle wall. Taken together, these results suggest that light may improve early pineal development by promoting the proliferation of specific cells in pineal gland and its attendant morphological structure.
Additionally, environmental factors can also accelerate the maturation of pineal rhythmic function mainly by targeting components of intracellular oscillator [31]. The components of intracellular oscillator have demonstrated little variation among various cell types [32]. CLOCK and BMAL1 are circadian clock proteins, which heterodimerize and bind promoter E-box elements to activate transcription of cryptochrome $(C R Y)$ and period (PER) genes. Sufficiently high levels of $C R Y$ and PER lead their nuclear translocation and subsequent interaction with the heterodimer of CLOCK:BMAL1 to inhibit their transcription [33]. Knockdown of CLOCK has been shown to cause a decrease in AANAT activity, demonstrating that AANAT is also an essential component of the circadian clock [34]. In the present study, the expression of clock-related genes including BMAL1, CLOCK, and $C R Y 1$, as well AANAT exhibited significant circadian rhythm under the 12L:12D light stimulation, but not under the constant dark condition. In tandem with the result of the present study, Kommedal et al. [35] reported a clear $24 \mathrm{~h}$ rhythm in the expression of $A A N A T$ and CLOCK genes following 12L:12D light stimulation during embryogenesis; Archer et al. [36] linked 12L:12D photoperiod during incubation with a long-lasting effect 

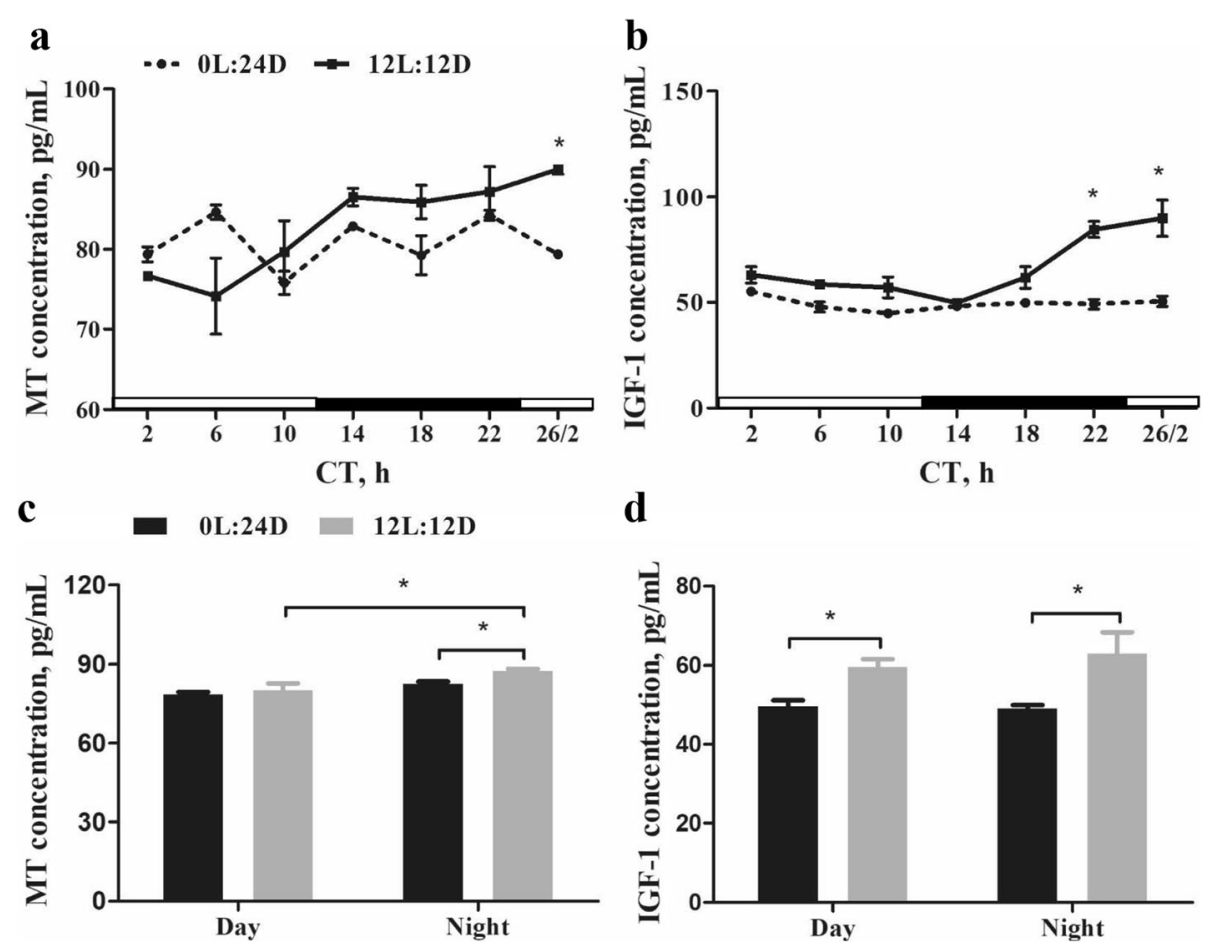

d

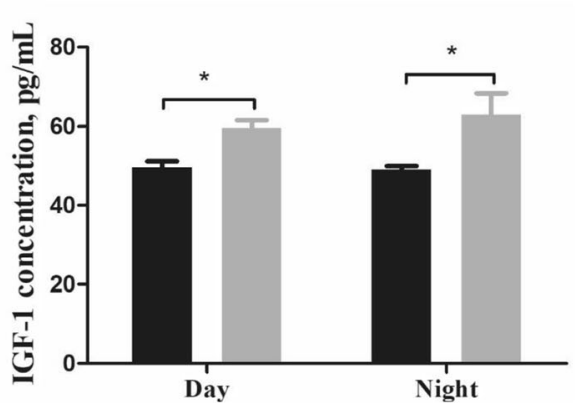

Fig. 6 The serum concentrations of MT and IGF-1. Line charts representing temporal changes of MT (a) and IGF-1 (b) levels. Histogram representing the concentrations of MT (c) and IGF-1 (d) at the subjective day and night in groups of OL:24D and 12L:12D. The horizontal white bar represents the subjective day, and the black bar represents the subjective night for the 12L:12D group. The results are presented as mean \pm SEM ( $n=3$ per group). Each column represents as mean \pm SEM. ${ }^{*} P<0.05$. CT, circadian time. Day, subjective day; Night, subjective day

on the diurnal rhythms of behavior, which may relate to the rhythmic expression of circadian clock components. These results indicate that light stimulation during incubation may accelerate the maturation of pineal function on circadian rhythm.

In this study, monochromatic green light stimulation promoted the secretion of IGF-1, which is mainly secreted by liver. The advantage on liver development was indeed observed in the 12L:12D group in this study. IGF-1 as a growth factor was reportedly involved in both the proliferation and differentiation of myoblasts [37], satellite cells [7] and pancreatic islets [38]. Previous studies reported elevated levels of thyroid hormone, IGF-1, and growth hormones and their association with either embryonic or post-hatch growth in broiler chickens [39, 40]. Lu et al. [41] suggested that IGF-1 may play important roles in accelerating embryo development. The liver, as the main organ for secreting IGF-1, plays a key role in fat storage absorbed from the yolk at the later stages of embryonic development. It was reported that antioxidant status is a vital aspect of chicken embryo liver development [42]. Melatonin, the main product secreted by the pineal gland, is known as an effective antioxidant [43]. Its exogenous application improved antioxidant status of hepatocytes [11] and was also reported to promote the hepatocyte proliferation and IGF-1 secretion in vitro [44]. Following the observation of light-induced changes in morphological structure and rhythmic function of pineal gland, we hypothesized that the MT concentration may be perturbed. Accordingly, results showed elevated serum MT concentration in embryos incubated under light stimulation during subjective night. Interestingly, we observed a consistent trend of plasma MT levels with the rhythmic expression of AANAT in this study. Both MT concentration and AANAT expression were high during subjective night, but relatively lower during subjective day period. This suggests that MT may play a central role in the development of chick embryo by promoting proliferation of hepatocytes (heavier liver) and consequent upsurge in IGF-1 secretion. However, further research is needed to determine whether the increase in MT concentration, its rhythmic secretion, or both as the cause of the elevated serum IGF-1. Nevertheless, pineal gland is suggested to be the key responsive organ in the lighting process that indirectly regulates embryo development through the increase in the synthesis and secretion of IGF-1 in chicks' liver via secretion of MT. 


\section{Conclusions}

Our results here indicate that the 12L:12D monochromatic green light stimulation shortens the hatching time without impairing hatching performance. Early pineal development was promoted by the 12L:12D monochromatic green light stimulation, as evidenced by modulating histological structure (including follicle area, wall thickness and lumen area) and accelerated maturation of rhythmic function. This further promote the secretion of MT with consequent increase in IGF-1 secretion in livers, which promotes subsequent embryonic development and shortening of the hatching time.

\section{Supplementary Information}

The online version contains supplementary material available at https://doi. org/10.1186/s40104-020-00539-x.

Additional file 1: Table S1. Primer sequences for qRT-PCR assays of target and reference genes.

Additional file 2: Fig. S1. Incubator temperature over d 7 post incubation of two incubators of each group.

\section{Abbreviations}

LED: Light emitting diode; IGF-1: Insulin-like growth factor 1; BMAL1: Brain and muscle ARNT-like-1; CLOCK: Circadian locomotor output cycles kaput; CRY1: Cryptochrome-1; AANAT: Arylalkylamine N-acetyltransferase; FL: Follicle; LM: Lumen; WT: Wall thickness; ZT: Zeitgeber time; GAPDH: Glyceraldehyde3-phosphate dehydrogenase; MT: Melatonin; CT: Circadian time; CRY: Cryptochrome; PER: Period

\section{Acknowledgments}

Many thanks to Chao Chen and Huanlin Chen (Institute of Animal Sciences of Chinese Academy of Agricultural Sciences, China) for the animal care, eggs collection, and incubation management.

\section{Authors' contributions}

CJ, LX, SY, and WP designed the experiments. WP and SY analyzed the data and wrote the manuscript. WP, LY, FJ, ZY, IAM, SL, WY, GP, JL, BS, and MH performed the phenotype and sample collection. $Y Z$ provided technical assistance for photo-incubation. All authors submitted comments on the draft, read, and approved the final manuscript.

\section{Funding}

Financial support of this study was provided by The National Key Research and Development Program of China (grant number 2016YFD0500502), China Agriculture Research Systems (grant number CARS-40), Fundamental Research Funds for Central Non-profit Scientific Institution (grant number 2018YWF-YB-20), and Agricultural Science and Technology Innovation Program (grant number ASTIP-IAS04).

\section{Availability of data and materials}

The data supporting the conclusions of this article is included within the article and its additional file.

\section{Ethics approval and consent to participate}

All experimental procedures for this research were performed according to local ethical guidelines and met the requirements of the animal care and use committee (No. IAS2020-14) of the Institute of Animal Sciences, Chinese Academy of Agricultural Sciences.

\section{Consent for publication}

Not applicable.

\section{Competing interests}

The authors declare that they have no competing interests.

\section{Author details}

'Institute of Animal Sciences, Chinese Academy of Agricultural Sciences, Key Laboratory of Animal (Poultry) Genetics Breeding and Reproduction, Ministry of Agriculture and Rural Affairs, Beijing 100193, China. ${ }^{2}$ College of Animal

Science and Technology, Northwest A\&F University, Yangling 712100, China. ${ }^{3}$ Beijing DQY Agricultural Science and Technology Co., Ltd., Beijing 100094, China.

Received: 21 June 2020 Accepted: 14 December 2020

Published online: 02 February 2021

\section{References}

1. Prescott N, Wathes C. Spectral sensitivity of the domestic fowl (Gallus g. domesticus). Brit Poult Sci. 2010;40(3):332-9.

2. Lewis PD, Morris TR. Poultry and coloured light. World Poult Sci. 2000;56(3): 189-207.

3. Foster RG, Follett BK. The involvement of a rhodopsin-like photopigment in the photoperiodic response of the Japanese quail. J Comp Physiol A. 1985; 157(4):519-28.

4. Rozenboim I, Piestun Y, Mobarkey N, Barak M, Hoyzman A, Halevy O. Monochromatic light stimuli during embryogenesis enhance embryo development and posthatch growth. Poult Sci. 2004;83(8):1413-9.

5. Zhang L, Zhang H, Qiao X, Yue H, Wu S, Yao J, et al. Effect of monochromatic light stimuli during embryogenesis on muscular growth, chemical composition, and meat quality of breast muscle in male broilers. Poult Sci. 2012;91(4):1026-31.

6. Xie D, Wang Z, Cao J, Dong Y, Chen Y. Effects of monochromatic light on proliferation response of splencyte in broilers. Anat Histol Embryol. 2008; 37(5):332-7

7. Liu W, Wang Z, Chen YJAR. Effects of monochromatic light on developmental changes in satellite cell population of pectoral muscle in broilers during early posthatch period. Anat Rec. 2010;293(8):1315-24.

8. Yeager RL, Franzosa JA, Millsap DS, Angell-Yeager JL, Heise SS, Wakhungu P, et al. Effects of 670-nm phototherapy on development. Photomed Laser Surg. 2005;23(3):268-72.

9. Tong Q, McGonnell IM, Demmers TGM, Roulston N, Bergoug H, Romanini $\mathrm{CE}$, et al. Effect of a photoperiodic green light programme during incubation on embryo development and hatch process. Animal. 2018;12(4): 765-73.

10. Halevy O, Piestun Y, Rozenboim I, Yablonka-Reuveni Z. In ovo exposure to monochromatic green light promotes skeletal muscle cell proliferation and affects myofiber growth in posthatch chicks. Am J Physiol Regul Integr Comp Physiol. 2006;290(4):R1062-70.

11. Li S, Cao J, Wang Z, Dong Y, Wang W, Chen Y. Melatonin mediates monochromatic light-induced insulin-like growth factor 1 secretion of chick liver: involvement of membrane receptors. Photochem Photobiol. 2016; 92(4):595-603.

12. Rosen CJ, Pollak M. Circulating IGF-I: new perspectives for a new century. Trends in Endocrinol Metab. 1999;10(4):136-41.

13. Haldar C, Bishnupuri KS. Comparative view of pineal gland morphology of nocturnal and diurnal birds of tropical origin. Microsc Res Tech. 2001;53(1): 25-32.

14. Cuello AC, Tramezzani JH. The epiphysis cerebri of the Weddell seal: its remarkable size and glandular pattern. Gen Comp Endocrinol. 1969;12(1): 154-64.

15. Tan DX, Manchester LC, Sainz RM, Mayo JC, Josefa L, Reiter RJ. Physiological ischemia/reperfusion phenomena and their relation to endogenous melatonin production: an hypothesis. Endocrine. 2005;27(2):149-58.

16. Ma S, Wang Z, Cao J, Dong Y, Chen Y. BMAL1 but not CLOCK is associated with monochromatic green light-induced circadian rhythm of melatonin in chick pinealocytes. Endocr Connect. 2019;8(1):57-68.

17. Zhong Z, Yu Y, Jin S, Pan J. Effects of mixing eggs of different initial incubation time on the hatching pattern, chick embryonic development and post-hatch performance. Peer J. 2018;10(6):e4634.

18. Careghi C, Tona K, Onagbesan O, Buyse J, Decuypere E, Bruggeman V. The effects of the spread of hatch and interaction with delayed feed access after hatch on broiler performance until seven days of age. Poult Sci. 2005; 84(8):1314-20.

19. Tona K, Bamelis F, Ketelaere BD, Bruggeman V, Moraes VMB, Buyse J, et al. Effects of egg storage time on spread of hatch, chick quality, and chick juvenile growth. Poult Sci. 2003;82(5):736-41. 
20. Livak KJ, Schmittgen TD. Analysis of relative gene expression data using realtime quantitative PCR and the 2(-Delta Delta C(T)). Methods. 2001;25(4):402-8.

21. Singh D, Rani S, Kumar V. Daily expression of six clock genes in central and peripheral tissues of a night-migratory songbird: evidence for tissue-specific circadian timing. Chronobiol Int. 2013;30(10):1208-17.

22. Lotvedt $P$, Jensen $P$. Effects of hatching time on behavior and weight development of chickens. PLoS One. 2014;9(7):e103040.

23. Yael N, David S. Posthatch development in poultry. J Appl Poult Res. 1997; 6(3):344-54

24. Dibner JJ, Knight CD, Kitchell ML, Atwell CA, Downs AC, Lvey F. Early feeding and development of the immune system in neonatal poultry. J Appl poultry Res. 1998;7(4):425-36.

25. Gaglo-Disse A, Tona K, Aliou S, Debonne M, Aklikokou K, Gbeassor M, et al. Effect of delayed feed access on production and blood parameters of layertype chicks. Acta Vet Hung. 2010;58(2):211-9.

26. Hulet R, Gladys G, Hill D, Meijerhof R, El-Shiekh T. Influence of egg shell embryonic incubation temperature and broiler breeder flock age on posthatch growth performance and carcass characteristics. Poult Sci. 2007; 86(2):408-12.

27. Boya J, Calvo J. Ultrastructural study of the post-hatching evolution of the pineal gland of the chicken (Gallus gallus). Acta Anat (Basel). 1980;107(2): 143-68.

28. Calvo J, Boya J. Embryonic development of the pineal gland of the chicken (Gallus gallus). Cells Tissues Organs. 1978;101(4):289-303.

29. Boya J, Calvo J. Post-hatching evolution of the pineal gland of the chicken. Cells Tissues Organs. 1978;101(1):1-9.

30. Petrusewicz-Kosińska M, Przybylska-Gornowicz B, Ziółkowska N, Martyniuk K, Lewczuk B. Developmental morphology of the Turkey pineal organ. Immunocytochemical and ultrastructural studies. Micron. 2019:122:8-20.

31. Csernus VJ, Nagy AD, Faluhelyi N. Development of the rhythmic melatonin secretion in the embryonic chicken pineal gland. Gen Comp Endocrinol. 2007;152(2):148-53.

32. Lowrey PL, Takahashi JS. Mammalian circadian biology: elucidating genome-wide levels of temporal organization. Annu Rev Genomics Hum Genet. 2004;5(1):407-41.

33. Hsieh PN, Zhang L, Jain MK. Coordination of cardiac rhythmic output and circadian metabolic regulation in the heart. Cell Mol Life Sci. 2017; 75(3):403-16.

34. Haque R, Ali FG, Biscoglia R, Abey J, Weller J, Klein D, et al. CLOCK and NPAS2 have overlapping roles in the circadian oscillation of arylalkylamine $\mathrm{N}$-acetyltransferase mRNA in chicken cone photoreceptors. J Neurochem. 2010;113(5):1296-306.

35. Kommedal S, Csernus V, Nagy AD. The embryonic pineal gland of the chicken as a model for experimental jet lag. Gen Comp Endocrinol. 2013; 188:226-31.

36. Archer GS, Mench JA. The effects of the duration and onset of light stimulation during incubation on the behavior, plasma melatonin levels, and productivity of broiler chickens. J Anim Sci. 2014;92(4):1753-8.

37. Yu M, Wang H, Xu Y, Yu D, Li D, Liu X, et al. Insulin-like growth factor-1 (IGF1) promotes myoblast proliferation and skeletal muscle growth of embryonic chickens via the PI3K/Akt signalling pathway. Cell Biol Int. 2015; 39(8):910-22.

38. Picinato MC, Hirata AE, Cipolla-Neto J, Curi R, Carvalho CRO, Anhe GF, et al. Activation of insulin and IGF-1 signaling pathways by melatonin through MT1 receptor in isolated rat pancreatic islets. J Pineal Res. 2008;44(1):88-94.

39. McNabb FM. The hypothalamic-pituitary-thyroid (HPT) axis in birds and its role in bird development and reproduction. Crit Rev Toxicol. 2007;37(1-2): 163-93.

40. Zhang L, Zhang HJ, Wang J, Wu SG, Qiao X, Yue HY, et al. Stimulation with monochromatic green light during incubation alters satellite cell mitotic activity and gene expression in relation to embryonic and posthatch muscle growth of broiler chickens. Animal. 2014;8(01):86-93.

41. Lu JW, Mcmurtry JP, Coon CN. Developmental changes of plasma insulin, glucagon, insulin-like growth factors, thyroid hormones, and glucose concentrations in chick embryos and hatched chicks. Poult Sci. 2007;86(4): 673-83.

42. Yang S, Wang L, Shi Z, Ou X, Wang W, Chen X, et al. Transcriptional profiling of liver tissues in chicken embryo at day 16 and 20 using RNA sequencing reveals differential antioxidant enzyme activity. PLoS One. 2018; 13(2):e0192253.
43. Li XX, Yang XG, Lu YQ, Lu SS, Zhang M, Yao HI, et al. Protective effects of melatonin against oxidative stress in flow cytometry-sorted buffalo sperm. Reprod Domest Anim. 2012;47(2):299-307.

44. Wang $T$, Wang Z, Cao J, Dong Y, Chen Y. Monochromatic light affects the development of chick embryo liver via an anti-oxidation pathway involving melatonin and the melatonin receptor Mel1c. Can J Anim Sci. 2014;94(3): 391-400.

\section{Ready to submit your research? Choose BMC and benefit from:}

- fast, convenient online submission

- thorough peer review by experienced researchers in your field

- rapid publication on acceptance

- support for research data, including large and complex data types

- gold Open Access which fosters wider collaboration and increased citations

- maximum visibility for your research: over $100 \mathrm{M}$ website views per year

At BMC, research is always in progress.

Learn more biomedcentral.com/submissions 